\title{
L'approche interactionnelle en didactique des langues
}

\author{
Préparé par : \\ Mohamed Hassan Fikry Bakhit \\ Maître Assistant au Département de Curricula et de Méthodologie \\ Faculté de Pédagogie de Quéna \\ Université du sud de la vallée \\ m.fikry85@yahoo.com
}




\title{
L'approche interactionnelle en didactique des langues
}

\author{
Préparé par : \\ Mohamed Hassan Fikry Bakhit \\ Maître Assistant au Département de Curricula et de Méthodologie \\ Faculté de Pédagogie de Quéna \\ Université du sud de la vallée \\ m.fikry85@yahoo.com
}

\section{Résumé :}

Cet article se donne comme objectif de mettre en évidence l'importance de l'utilisation de l'approche interactionnelle en didactique des langues. La didactique des langues vivantes fait entrer dans une nouvelle ère avec l'émergence de cette approche. L'idée principale de l'approche interactionnelle est de développer la capacité de l'apprenant à utiliser la langue afin d'accomplir des tâches réalistes liées directement aux situations de la vie quotidienne dans des interactions sociales réelles dépassent le cadre de la classe. Cette approche aide l'apprenant à apprendre et acquérir en classe et hors de classe des connaissances et des compétences communicatives langagières (compétences linguistiques, compétences sociolinguistiques et compétences pragmatiques). Ce sont les finalités majeures de l'apprentissage des langues.

Mots-clés : l'approche interactionnelle, la tâche, la communication, acteur social 


$$
\begin{aligned}
& \text { مدخل الاداء التفاعلي في تعلبـم اللغات } \\
& \text { إعــــــداد } \\
& \text { م .م / محمـد حسن فكرى بخيت } \\
& \text { مدرس مساعد بقسم المناهج وطرق التدريس }
\end{aligned}
$$

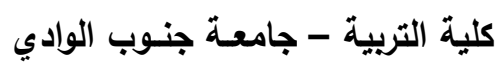

$$
\begin{aligned}
& \text { m.fikry85@yahoo.com }
\end{aligned}
$$

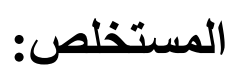

تهدف هذه المقالة الى تسليط الضوء على أهمية استخدام مدخل الاداء التفاعلي في تعليم اللغات، حيث ان تدريس اللغات الحية دَخل في حُقبة جديدة مع بزوغ هذا المدخل. ان الفكرة الرئيسية لهذا المدخل تقوم على أساس تتمية قدرة المتعلم على استخدام اللغة من اجل انجاز مهام واقعية مستمدة مباشرة من وقائع الحياة اليومية التي تقوم على التفاعل الاجتماعي الحقيقي الذى يتجاوز حدود الفصل الدراسي. وبستطيع المتعلم من خلال هذا المدخل تعلم واكتساب العديد من مهارات التواصل اللُغوي المختلفة سواء أكان هذا داخل قاعات الفصل الدراسي أو خارجها منل المهارات اللُغوية والمهارات اللُغوية الاجنماعية وكذلك المهارات العملية. وهذه هي الغاية القصوى من تعلم اللغات.

الكلمات المفتاحيةة: مدخل الاداء التفاعلي، المَهَمة، التو اصل، مُهَنَل اجتماعي 


\section{L'approche interactionnelle en didactique des langues \\ Mohamed Hassan Fikry Bakhit}

\section{Introduction :}

L'approche interactionnelle est considérée comme une des modalités modernes basées sur la création de nouvelles situations d'apprentissage exigeant l'utilisation des stratégies d'apprentissage actif, de la stratégie d'auto-apprentissage et d'apprentissage concentré sur l'apprenant. L'importance de cette approche est déterminée en augmentant l'efficacité de l'apprentissage car elle fournit aux étudiants tous les moyens de communication et d'interaction entre eux, que ce soit en binaire ou en petits groupes.

\section{1- Origine et idée de l'approche interactionnelle:}

C'est une approche vise à développer la capacité de l'apprenant à utiliser la langue dans la communication naturelle, que ce soit en interaction avec les autres, ou en partageant des idées et des informations afin d'atteindre les tâches ciblées.

L'approche interactionnelle reflète le passage du paradigme de la communication au paradigme de l'action. On ne communique plus seulement pour parler avec l'autre mais pour agir avec l'autre. Ceci est la base sur laquelle repose l'approche de la communication pour enseigner les langues étrangères.

Cette approche est basée sur le modèle fonctionnel de la langue et la théorie de la compétence communicative qui signifie " la capacité de l'individu à utiliser la langue automatiquement, avec un sens linguistique par lequel cet individu distingue entre les différentes fonctions de la langue dans les situations de communications réelles pour accomplir des tâches spécifiques".

De leur part, Lions-Olivieri et Liria (2009) montrent que l'idée de l'approche interactionnelle vient $d u$ fait que la langue n'est pas simplement un système de règles, mais une source dynamique pour créer des significations, donc la connaissance de la langue seule ne suffit pas à apprendre, mais doit être appliquée de manière communicative pour atteindre la langage fonctionnel; où cette approche vise à développer l'efficacité de la communication chez les étudiants.

Selon l'approche interactionnelle, l'apprenant est aussi apprenant-usager d'une langue, que les tâches communicatives ne sont pas seulement langagières, que les actes de parole sont aussi 
des actes sociaux, et que l'on peut dire que tout apprenant est un acteur social.

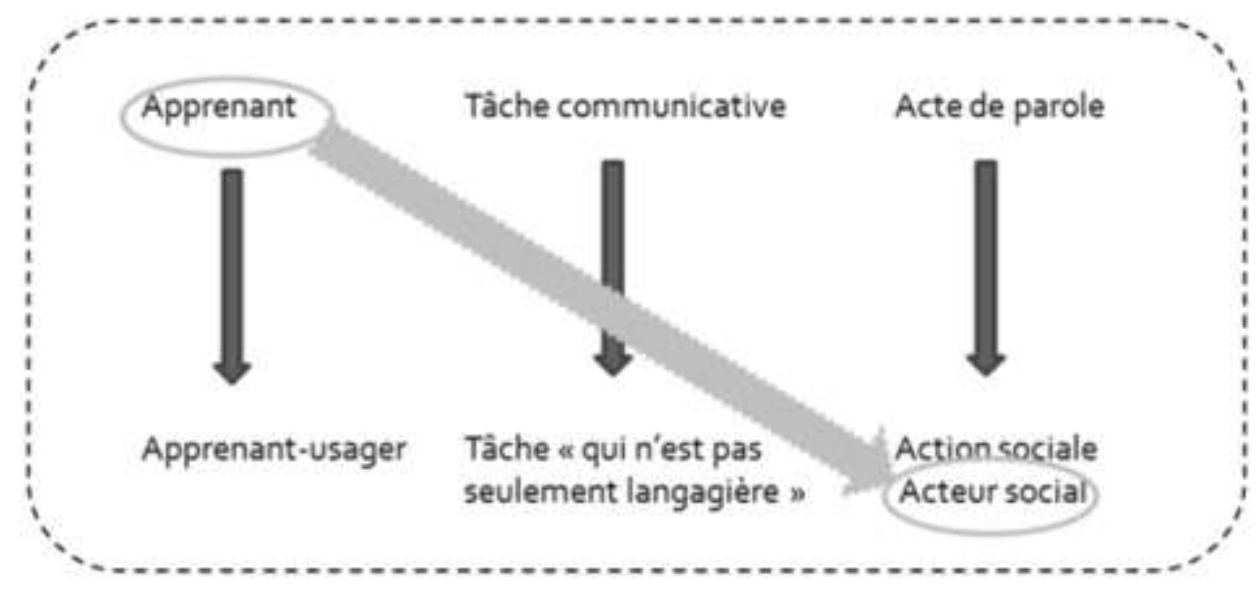

Figure $\mathrm{N}^{\circ}(1)$ : L'apprenant devenu acteur social

L'approche interactionnelle est une façon différente d'apprendre une langue. Elle peut aider l'étudiant (e) en le/la plaçant dans une situation identique au monde réel; une situation dans laquelle la communication orale est essentielle pour accomplir une tâche précise.

\section{2- La conception de 1'approche interactionnelle :}

Le Cadre Européen Commun de Références pour les langues (appelé ci-après CECR) propose une vision de la communication comme action où le locuteur est considéré comme un acteur social.

Le CECR privilégie dans l'apprentissage, l'enseignement et l'évaluation des langues vivantes une perspective interactionnelle : La perspective privilégiée ici est, très généralement aussi, de type interactionnel en ce qu'elle considère avant tout l'usager et l'apprenant d'une langue comme des acteurs sociaux ayant à accomplir des tâches (qui ne sont pas seulement langagières) dans des circonstances et un environnement donnés, à l'intérieur d'un domaine d'action particulier. Si les actes de parole se réalisent dans des activités langagières, celles-ci s'inscrivent elles-mêmes à l'intérieur d'actions en contexte social qui seules leur donnent leur pleine signification (CECR, $2001: 15)$. 


\section{L'approche interactionnelle en didactique des langues \\ Mohamed Hassan Fikry Bakhit}

Pour Bange, Carol et Griggs (2005), l'apprentissage se fait toujours dans un cadre socio-cognitif. En effet, "cognition et interaction sont inséparables "

De sa part, Ollivier (2012) a défini l'approche interactionnelle comme une approche didactique s'inscrivant dans les théories linguistiques et pédagogiques qui mettent les interactions sociales en avant, lesquelles forment, à la fois, le cadre et un élément constitutif déterminants des actions et interactions humaines. L'approche interactionnelle place ainsi les interactions sociales au cœur de ses préoccupations et implique de proposer aux apprenants des tâches à réaliser au sein d'interactions sociales et se concentrer sur la réalisation sociale de la tâche tout autant, voire plus que sur l'apprentissage de la langue.

Egalement, Saydi (2015) a souligné que l'approche interactionnelle adopte une technique qui oriente l'apprenant à être plutôt acteur social : l'apprenant est encouragé à utiliser la langue d'apprentissage dans la société même, afin de réaliser une action, résoudre une situation problématique ou favoriser un fait.

Quant à Zhihong (2011), l'approche interactionnelle vise à développer un ensemble de compétences. Apprendre une langue, c'est apprendre et acquérir en classe et hors de classe des connaissances et compétences liées directement à la vie, puis les réutiliser dans la vie (en continuant à apprendre et à se perfectionner). Ces compétences concernent les compétences générales (savoirs, aptitudes et savoir-faire, savoir-être ainsi que savoir-apprendre).

Donc, on peut dire que l'approche interactionnelle a l'avantage de permettre à l'étudiant (e) d'utiliser ses capacités à son propre niveau développant ainsi son langage à travers l'utilisation de la langue. Elle a également l'avantage d'aider l'étudiant (e) à se concentrer sur les buts à atteindre de telle sorte que le langage devient un outil et enfin, l'avantage de transformer l'usage de la langue en nécessité. 


\section{L'approche interactionnelle en didactique des langues \\ Mohamed Hassan Fikry Bakhit}

\section{3- Critères de l'approche interactionnelle:}

Il y a un certain nombre de critères que nous nous pouvons organiser en vue de décrire l'approche interactionnelle de l'apprentissage/enseignement des langues étrangères.

De sa part, Rodriguez (2013) explique huit critères principales sur ce sujet:

1- L'approche interactionnelle incite à la communication entre les apprenants afin d'accomplir une tâche.

2- Les étudiants évoluent librement et choisissent la langue qu'ils utiliseront en fonction de leurs besoins.

3- Il faut qu'elle soit réalisée en coopération.

4- C'est un plan de travail centré sur le sens : la langue est un outil pour atteindre un objectif, et non un objectif en soi.

5- Elle implique la mise en place de processus réels d'utilisation de la langue: il s'agit de se faire comprendre, non réciter des listes de vocabulaire.

6- Elle peut impliquer le recours à n'importe quelle compétence linguistique, ou à toutes à la fois.

7- Elle doit mettre en place des processus cognitifs complexes.

8- Elle aboutit à un produit final.

4- Importance de l'utilisation de l'approche interactionnelle dans l'apprentissage/enseignement:

L'utilisation de l'approche interactionnelle dans l'apprentissage/ enseignement a plusieurs avantages et caractéristiques positives.

Griggs (2009) a identifié l'importance de cette approche dans les points suivants :

1- L'intérêt de cette approche est que l'apprenant est placé au cœur de la réflexion, c'est lui qui va développer des stratégies de résolutions de problèmes afin d'optimiser ses ressources langagières et progresser vers son autonomie.

2- Contribuer à l'amélioration des processus de compréhension chez les étudiants ordinaires et les étudiants ayant des difficultés d'apprentissage.

3- Augmenter l'attention et la concentration des étudiants lors de l'exécution de tâches. 


\section{L'approche interactionnelle en didactique des langues}

Mohamed Hassan Fikry Bakhit

4- Contribuer à l'acquisition de concepts et de compétences linguistiques facilement.

5-Augmenter la motivation des étudiants et leur désir de lire et encourager les étudiants faibles à participer.

6- Soutenir la confiance des étudiants en eux-mêmes et le sentiment d'accomplissement chez eux.

7- Développer la capacité des étudiants à contrôler leurs processus de réflexion.

8- Offrir aux étudiants l'occasion de s'engager dans des activités d'apprentissage et de découverte.

9- Fournir des rétroactions et renforcer les réponses des étudiants.

10- Fournir un environnement d'apprentissage plus riche qui ne dépend pas d'une méthode seulement.

\section{5- Avantages de 1'utilisation de l'approche interactionnelle dans l'apprentissage/enseignement des langues:}

L'approche interactionnelle fournit des solutions pratiques et scientifiques aux problèmes d'apprentissage des langues.

Arditty (2005) met en évidence que l'utilisation de l'approche interactionnelle dans le processus de l'apprentissage/enseignement réalise de nombreux avantages, ce sont:

1- L'approche interactionnelle est utile pour mettre en avant l'étudiant dans le processus d'apprentissage au lieu du professeur. 2- Elle offre à l'étudiant une façon différente de percevoir la langue comme un outil plutôt qu'un but à atteindre.

3- L'approche interactionnelle offre une opportunité de communication entre les apprenants dans un contexte social vivant.

4- Elle peut faire l'apprentissage passer d'un savoir abstrait à une application dans le monde réel.

5- L'approche interactionnelle donne à l'apprenant une opportunité suffisante pour pratiquer la nouvelle tâche sous la supervision de l'enseignant.

6- La tâche est utile car elle s'adresse aux besoins immédiats des apprenants et propose un cadre pour créer des leçons qui sont intéressantes et qui répondent aux besoins des étudiants.

7- Elle offre aux professeurs une nouvelle opportunité de diversifier leurs stratégies d'enseignement. 


\section{L'approche interactionnelle en didactique des langues \\ Mohamed Hassan Fikry Bakhit}

8- Elle fournit un environnement d'apprentissage approprié dans lequel l'enseignant, l'apprenant, le matériel d'apprentissage, et les ressources disponibles dans la salle de classe interagissent.

9- Elle aide à surmonter la réticence des étudiants qui n'aiment pas les langues étrangères.

\section{6- La tâche dans l'approche interactionnelle:}

L'approche interactionnelle se base, comme son nom l'indique, sur l'action autrement dit sur la tâche.

Da sa part, Nunan (2000) a défini la tâche comme une unité de travail centrée sur le sens impliquant les apprenants dans la compréhension, la production et/ou l'interaction en langue cible.

Selon le CECR (2001 :121), la tâche est un ensemble d'actions finalisées dans un certain domaine avec un but défini et un produit particulier. Tâche et action ne sont donc pas équivalentes mais complémentaires. La tâche est hyponyme de l'action: elle est comprise dans l'action. Elle est en aussi le vecteur puisqu'elle est à son service.

De sa part, Puren (2006) définit la tâche ainsi "unité de sens dans l'activité d'enseignement-apprentissage". Le concept de tâche correspond en fait à l'agir d'apprentissage.

Puren réserve le terme $\mathrm{d}^{\prime}$ « action » pour l'agir d'usage ou l'agir social.

Pour Branden (2006), la tâche est une activité menée par une personne pour atteindre un objectif particulier et doit utiliser la langue.

Selon Coste (2009), la notion de tâche renvoie à une action finalisée, avec un début, un achèvement visé, des conditions d'effectuation, des résultats constatables.

7- Caractéristiques de la tâche dans l'approche interactionnelle:

Il existe un ensemble de caractéristiques qu'on peut prendre en compte pendant la préparation et la construction des tâches dans des situations éducatives.

Selon Nunan (1991), cinq caractéristiques marquent l'enseignement par tâches :

1- L'accent mis sur l'apprendre à communiquer au travers des activités d'échanges interactives en langue cible ; 


\section{L'approche interactionnelle en didactique des langues \\ Mohamed Hassan Fikry Bakhit}

2- L'introduction des textes authentiques dans la situation d'apprentissage ;

3- Les apprenants se concentrent non seulement sur la langue, mais aussi sur le processus de l'apprentissage ;

4- Une amélioration des expériences personnelles de l'apprenant comme éléments importants contribuant à l'apprentissage en classe ; 5- Une tentative de lier l'apprentissage de la langue en classe avec sa réutilisation en dehors de la salle de classe.

Egalement, Perrichon (2009) a dessiné cinq caractéristiques principales de la tâche :

1 - le sens est fondamental.

2- déterminer le rôle de chaque apprenant dans l'accomplissement de la tâche.

3- la tâche a une relation avec des attitudes réalistes à l'égard de l'utilisation de la langue.

4- priorité pour compléter la tâche.

5- la tâche est évaluée à la lumière de l'issue.

8- Typologies des tâches dans l'approche interactionnelle:

De leur part, Chaudron et Valcárcel (1999) ont spécifié six catégories de tâches:

1- tâches contrôlées par l'enseignant (p. ex.: lecture à haute voix, jeu de questions-réponses, exercice, traduction, dictée, explication d'un contenu, copier, reconnaître, identifier, réviser),

2- tâches semi-contrôlées (p. ex.: dialogue, échange d'informations, jeu de questions-réponses, raconter une histoire, brainstorming),

3- tâches libres (p. ex.: jeu de rôle, simulation, discussion, résolution de problème, jeux, rédaction, faire du théâtre).

4- tâche consistant à transmettre une information,

5- tâche consistant à prendre une information nouvelle à partir d'informations données,

6- tâche consistant à donner une opinion.

Egalement, Candlin et Nunan (2001) ont souligné quatre types de tâches:

1- tâches focalisées sur la reconnaissance et la perception,

2- tâches focalisées sur la sélection et la classification,

3- tâches focalisées sur l'inférence et la déduction,

4- tâches focalisées sur le transfert et la généralisation. 


\section{9- Phases d'exécution de la tâche dans l'approche interactionnelle:}

L'exécution de la tâche dans le cours d'apprentissage de la langue passe par trois étapes principales: préparation de la tâche, réalisation de la tâche et post-tâche. Voici quelques détails de ces étapes (Gilbert, 2007) :

1- La phase de la préparation de la tâche :

C'est la phase préparatoire. Cette phase vise à préparer les apprenants pour accomplir la tâche d'une manière qui les motive à travailler et les aide à compléter la tâche avec succès.

2- La phase de la tâche elle-même :

$C^{\prime}$ est la phase pendant laquelle un travail de réalisation doit avoir lieu. A cette phase, les apprenants mènent sous forme binaire ou en petits groupes la tâche requise, en mettant l'accent sur la signification et pour rechercher l'issue souhaitée.

Pendant cette phase, les apprenants produisent, jouent et présentent leurs tâches par exemple :

- en produisant un poster.

- en jouant un jeu de rôle.

- en organisant un débat.

- en produisant une brochure.

- en faisant un exposé.

3- La phase de post-tâche :

Cette phase peut inclure trois choses, la première étant donnée aux étudiants l'occasion de refaire la tâche.

La deuxième chose est un rapport sur la tâche, dans lequel les apprenants résument l'issue finale qu'ils ont atteint et montrent comment ils accomplissent la tâche et les problèmes auxquels ils étaient confrontés, la façon de les surmonter.

La troisième chose est mis l'accent sur le matériel, afin de parvenir à une meilleure compréhension de la langue.

\section{0- Rôle de l'enseignant dans l'approche interactionnelle:}

L'enseignant joue plusieurs rôles dans l'approche interactionnelle pour apprendre et enseigner la langue.

De sa part, Romainville (2001) a présenté les rôles de l'enseignant les plus importants dans cette approche comme :

- Sélectionner et organiser les tâches. 
- Préparer les apprenants à effectuer la tâche.

-Attirer l'attention des apprenants à la formule de langue cible.

-Entraîner les apprenants sur les stratégies nécessaires pour accomplir la tâche.

- Orienter les apprenants au cours de l'exécution de la tâche.

- Motiver et encourager les apprenants à accomplir la tâche.

- Fournir l'assistance au besoin.

11- Rôle de l'apprenant dans l'approche interactionnelle:

L'apprentissage et l'enseignement des langue basé sur l'approche interactionnelle sont des approches centrées sur l'apprenant.

Clementina et al (2011) ont mentionné que l'apprenant :

- est actif ; acteur social ;

- agit en co-action avec les autres ;

- réalise des actes, y compris de parole ;

- a un objectif social de référence qui dépasse la situation d'enseignement-apprentissage ;

- surmonte les obstacles langagiers, affectifs, sociaux et pragmatiques ;

- transforme les verbes d' "agir, réagir et interagir "à des "gestes" nécessaires à l'apprentissage de la compétence communicative. 


\section{L'approche interactionnelle en didactique des langues \\ Mohamed Hassan Fikry Bakhit}

\section{Bibliographie:}

Arditty, J. (2005) .Approches interactionnistes : exemples de fondements théoriques et questions de recherche », Le français dans le monde, Recherches et applications, numéro spécial, juillet 2005, p. 8-19.

Bange, P., Carol, R., Griggs \& Peter. (2005). L'apprentissage d'une langue étrangère : Cognition et interaction. Paris: Le Harmattan.

Branden, K. (2006). Introduction: Task-Based Language Teaching in a Nutshell In: K. Branden (ed.) Task-Based Language Education. Cambridge: Cambridge University Press.

Candlin, C., \& Nunan, D. (2001). Revised Syllabus Specifications for the Omani School English Language Curriculum. Muscat Ministry of Education and Youth.

Chaudron, C., \& Valcárcel, M. (1999). A process-product study of communicative language teaching (Final report submitted to the Comite Conjunto Hispano Norteamericano para la Cooperación Cultural y Educativa, Madrid). Murcia : Universidad de Murcia, Escuela Universitaria de Magisterio.

Clementina, A., Anghel, M., Dobre, C., Groza, D., Mathe, M., Popescu, C.\& Zoicas,M. (2011). La formation contenue des enseignants à l'utilisation des instruments informatiques modernes dans l'enseignement efficace du français et l'évaluation au niveau européen des compétences linguistiques. Manuel de formation. Projet Ministère de l'Education, de la Recherche, de la Jeunesse et du Sport.

Disponible

sur :

http//flewebpedagogique.com/.../files/.../Manue 1- Module2.do...

Conseil de l'Europe. (2001). Cadre européen commun de référence pour les langues : Apprendre, enseigner, évaluer. Paris : Les Éditions Didier.

Coste, D. (2009). Tâche, progression, curriculum . Le français dans le monde : recherches et applications, $\mathrm{n}^{\circ} 45, \mathrm{p} .15-24$.

Gilbert, R. (2007). Effects of manipulating task complexity on selfrepairs during L2 oral production. International Review of Applied Linguistics in Language Teaching, 45 (3), 215-240.

Griggs, P. (2009). A propos de l'articulation entre l'agir de l'usage et l'agir de l'apprentissage dans une approche actionnelle : une 


\section{L'approche interactionnelle en didactique des langues \\ Mohamed Hassan Fikry Bakhit}

perspective sociocognitive. In : L'approche actionnelle dans l'enseignement des langues. Paris : Edition Maison des langues, 2 ème édition révisée et enrichie, pp.79-100.

Lions-Olivieri, M-L. \& Liria, P. (2009). L'approche actionnelle dans l'enseignement des langues onze articles pour mieux comprendre et faire le point. Paris : Éditions Maison des langues.

Nunan, D. (1991). Communicative tasks and language curriculum . TESOL Quarterly, n²5, pp. 279-295.

Nunan, D. (2000). Designing Tasks for the Communicative Classroom. Cambridge : Cambridge University Press.

Ollivier, C. (2012). Approche interactionnelle et didactique invisibleDeux concepts pour la conception et la mise en œuvre de tâches sur le web social. Alsic [En ligne], Vol. 15, n 1 . Consulté le 30 septembre 2017 . Disponible sur : http://alsic.revues.org/2402

Perrichon, É. (2009). Perspective actionnelle et pédagogie du projet : De la culture individuelle à la construction d'une culture d'action collective, Synergies des Pays Riverains de la Baltique, n 6, 91-111. Disponible sur : www.gerfli nt .fr /Base/Baltique6/perrichon.pdf

Puren, C. (2006). Comment harmoniser le système d'évaluation français avec le cadre européen commun de référence? Communication présentée à la Conférence de l'Assemblée Générale de l'APLV Régionale de Grenoble, France.

Rodriguez, A, S. (2013). L'approche par les tâche. Disponible sur l'adresse suivant: http://www.ocw.um.es/cc.-soci ales /...en.../1app roche-par-les-taches.pdf

Romainville, M. (2001). Les implications didactiques de l'approche par les compétences, in «Enjeux» n51/52, Service de pédagogie universitaire, FUND.

Saydi, T. (2015). L'approche actionnelle et ses particularités en comparaison avec l'approche communicative. Synergies Turquie $n^{\circ}$ 8 p. 13-28.

Zhihong, P. (2011). La perspective actionnelle par tâches et la culture $\mathrm{d}^{\prime}$ apprentissage chinois. Synergies Chine $\mathrm{n}^{\circ} 6-\mathrm{pp} .37-45$. 